\title{
Combinatorial congruences and Stirling numbers
}

\author{
by \\ ZHI-WeI Sun (Nanjing)
}

1. Introduction. In a recent paper of the author and D. M. Davis [SD] originally motivated by the study of homotopy exponents of the special unitary group $\mathrm{SU}(n)$, the following theorem was established.

Theorem 1.0 (Sun and Davis). Let $p$ be a prime, and let $\alpha, n \in \mathbb{N}=$ $\{0,1, \ldots\}$ and $r \in \mathbb{Z}$. Then, for any $f(x) \in \mathbb{Z}[x]$, we have

$$
\begin{aligned}
\operatorname{ord}_{p}\left(\sum_{k \equiv r\left(\bmod p^{\alpha}\right)}(-1)^{k}\left(\begin{array}{l}
n \\
k
\end{array}\right) f\left(\frac{k-r}{p^{\alpha}}\right)\right) \\
\quad \geq \operatorname{ord}_{p}\left(\left\lfloor\frac{n}{p^{\alpha-1}}\right\rfloor !\right)-\operatorname{deg} f+\tau_{p}\left(\{r\}_{p^{\alpha-1}},\{n-r\}_{p^{\alpha-1}}\right),
\end{aligned}
$$

where $\operatorname{ord}_{p}(a)=\sup \left\{m \in \mathbb{N}: p^{m} \mid a\right\}$ is the p-adic order of $a \in \mathbb{Z},\{a\}_{p^{\alpha-1}}$ stands for the least nonnegative residue of a modulo $p^{\alpha-1}$ (and this is regarded as 0 if $\alpha=0)$, and for $a, b \in \mathbb{N}$ we use $\tau_{p}(a, b)$ to denote the number of carries occurring in the addition of $a$ and $b$ in base $p$.

Let $p$ be a prime. By a well-known fact in number theory (cf. [IR, p. 26]),

$$
\operatorname{ord}_{p}(n !)=\sum_{i=1}^{\infty}\left\lfloor\frac{n}{p^{i}}\right\rfloor \quad \text { for every } n=0,1,2, \ldots
$$

A useful theorem of E. Kummer asserts that if $a, b \in \mathbb{N}$ then

$$
\operatorname{ord}_{p}\left(\begin{array}{c}
a+b \\
a
\end{array}\right)=\sum_{i=1}^{\infty}\left(\left\lfloor\frac{a+b}{p^{i}}\right\rfloor-\left\lfloor\frac{a}{p^{i}}\right\rfloor-\left\lfloor\frac{b}{p^{i}}\right\rfloor\right)=\tau_{p}(a, b) .
$$

In this paper we will apply Theorem 1.0 to deduce three theorems on combinatorial congruences or Stirling numbers of the second kind.

2000 Mathematics Subject Classification: Primary 11B65; Secondary 05A10, 11A07, $11 \mathrm{~B} 73$.

Supported by the National Science Fund for Distinguished Young Scholars (Grant No. 10425103) and a Key Program of NSF (Grant No. 10331020) in China. 
For $l, m \in \mathbb{N}$ with $l+m>0$, the Stirling number $S(l, m)$ of the second kind is the number of ways to partition a set of cardinality $l$ into $m$ nonempty subsets; in addition, we define $S(0,0)$ to be 1 . It is well known that

$$
x^{l}=\sum_{j=0}^{l} S(l, j)(x)_{j} \quad \text { for } l=0,1,2, \ldots,
$$

where $(x)_{j}=\prod_{0 \leq i<j}(x-i)$ and an empty product has the value 1 (thus $\left.(x)_{0}=1\right)$.

Here is our first theorem.

Theorem 1.1. Let $p$ be any prime. Let $a \in \mathbb{Z}, l, l^{\prime}, m \in \mathbb{Z}^{+}=\{1,2, \ldots\}$, $l^{\prime} \geq l>m / p$ and

$$
l^{\prime} \equiv l\left(\bmod (p-1) p^{\left\lfloor\log _{p} m\right\rfloor-\delta_{p}(a, m)}\right),
$$

where

$$
\delta_{p}(a, m)= \begin{cases}1 & \text { if } a \in p \mathbb{Z} \text { and } \log _{p} m \in \mathbb{Z}^{+} \\ 0 & \text { otherwise. }\end{cases}
$$

Then

$$
\sum_{j=0}^{l^{\prime}}\left(\begin{array}{l}
l^{\prime} \\
j
\end{array}\right) S(j, m) a^{l^{\prime}-j} \equiv \sum_{j=0}^{l}\left(\begin{array}{l}
l \\
j
\end{array}\right) S(j, m) a^{l-j}(\bmod p) .
$$

Corollary 1.1. Let $p$ be a prime, and let $a \in \mathbb{Z}$ and $m \in \mathbb{Z}^{+}$. Then, for $k=m+(p-1) p^{\left\lfloor\log _{p} m\right\rfloor-\delta_{p}(a, m)} q$ with $q \in \mathbb{N}$, we have

$$
\sum_{j=0}^{k}\left(\begin{array}{l}
k \\
j
\end{array}\right) S(j, m) a^{k-j} \equiv 1(\bmod p) .
$$

Proof. Just apply Theorem 1.1 with $l=m$ and $l^{\prime}=k$.

REMARK 1.1. Note that if $p$ is a prime and $m$ is a positive integer then $m-(p-1) p^{\left\lfloor\log _{p} m\right\rfloor}<m / p$.

The following result was first obtained by L. Carlitz $[\mathrm{C}]$ in 1955. (See also A. Nijenhuis and H. S. Wilf [NW], and Y. H. H. Kwong [K].)

Corollary 1.2. Let $p$ be any prime. Suppose that $\alpha, m \in \mathbb{N}, m \geq p$ and $p^{\alpha}<m \leq p^{\alpha+1}$. Then $p^{\alpha}(p-1)$ is a period of the sequence $\{S(l, m)\}_{l \geq m}$ modulo $p$.

Proof. It suffices to apply Theorem 1.1 with $a=0$.

The sum $\sum_{k \equiv r(\bmod m)}\left(\begin{array}{l}n \\ k\end{array}\right)$ with $m \in \mathbb{Z}^{+}, n \in \mathbb{N}$ and $r \in \mathbb{Z}$ has been investigated intensively (see $[\mathrm{S}]$ for some historical background and related 
congruences). In 1899 J. W. L. Glaisher (cf. [D, p. 271] and [ST]) proved that

$$
\sum_{j \equiv r(\bmod p-1)}\left(\begin{array}{l}
l^{\prime} \\
j
\end{array}\right) \equiv \sum_{j \equiv r(\bmod p-1)}\left(\begin{array}{l}
l \\
j
\end{array}\right)(\bmod p)
$$

whenever $p$ is a prime, $r \in \mathbb{Z}, l^{\prime}, l \in \mathbb{Z}^{+}$and $l^{\prime} \equiv l(\bmod p-1)$. Clearly Glaisher's congruence is our result below in the case $m=1$.

Corollary 1.3. Let $p$ be a prime, $m \in \mathbb{Z}^{+}$and $r \in \mathbb{Z}$. For any $l^{\prime}, l \in \mathbb{Z}^{+}$ with $l^{\prime} \geq l>m / p$ and

$$
l^{\prime} \equiv l\left(\bmod (p-1) p^{\left\lfloor\log _{p} m\right\rfloor}\right),
$$

we have

$$
\sum_{j \equiv r(\bmod p-1)}\left(\begin{array}{l}
l^{\prime} \\
j
\end{array}\right) S(j, m) \equiv \sum_{j \equiv r(\bmod p-1)}\left(\begin{array}{l}
l \\
j
\end{array}\right) S(j, m)(\bmod p) .
$$

Our second theorem is slightly stronger than Conjecture 1.3 of the author and Davis $[\mathrm{SD}]$ which was proved in [SD] when $p=2$ and $r=0$.

Theorem 1.2. Let $p$ be a prime, and let $\alpha, l, n \in \mathbb{N}$ and $r \in \mathbb{Z}$. Set $r_{*}=\{r\}_{p^{\alpha}}, n_{*}=r_{*}+\{n-r\}_{p^{\alpha}}$ and

$$
m=\frac{n-n_{*}}{p^{\alpha}}=\left\lfloor\frac{r}{p^{\alpha}}\right\rfloor+\left\lfloor\frac{n-r}{p^{\alpha}}\right\rfloor .
$$

Suppose that $l \geq m>0$ and

$$
l \equiv m\left(\bmod (p-1) p^{\left\lfloor\log _{p} m\right\rfloor-\delta_{p}\left(\left\lfloor r / p^{\alpha}\right\rfloor, m\right)}\right),
$$

where the notation $\delta_{p}(a, m)$ is given by (1.2). Then

$$
\frac{1}{\left\lfloor n / p^{\alpha}\right\rfloor !\left(\begin{array}{c}
n_{*} \\
r_{*}
\end{array}\right)} \sum_{k \equiv r\left(\bmod p^{\alpha}\right)}(-1)^{k}\left(\begin{array}{l}
n \\
k
\end{array}\right)\left(\frac{k-r}{p^{\alpha}}\right)^{l} \equiv(-1)^{l+r_{*}}(\bmod p) .
$$

REmark 1.2. Theorem 1.2 implies that the inequality in Theorem 5.1 of $[\mathrm{DS}]$ is sharp for infinitely many values of $l$ provided that $n \geq 2 p^{\alpha}-1$.

Our third theorem confirms Conjecture 1.1 of [SD].

TheOREm 1.3. Let $p$ be any prime, and let $\alpha \in \mathbb{Z}^{+}, l, n \in \mathbb{N}$ and $r \in \mathbb{Z}$. Then

$$
\begin{aligned}
& \frac{1}{\left\lfloor n / p^{\alpha-1}\right\rfloor !} \sum_{k \equiv r\left(\bmod p^{\alpha}\right)}(-1)^{k}\left(\begin{array}{c}
p n \\
p k
\end{array}\right)\left(\frac{k-r}{p^{\alpha-1}}\right)^{l} \\
& \equiv \frac{1}{\left\lfloor n / p^{\alpha-1}\right\rfloor !} \sum_{k \equiv r\left(\bmod p^{\alpha}\right)}(-1)^{k}\left(\begin{array}{l}
n \\
k
\end{array}\right)\left(\frac{k-r}{p^{\alpha-1}}\right)^{l}\left(\bmod p^{a_{p}}\right),
\end{aligned}
$$


where

$$
a_{p}= \begin{cases}1 & \text { if } p=2, \\ 2 & \text { if } p=3 \\ 3 & \text { if } p>3\end{cases}
$$

REMARK 1.3. Let $p$ be a prime, $\alpha, n \in \mathbb{N}$ and $r \in \mathbb{Z}$. When $p^{\alpha}>n$ and $l=0 \leq r \leq n,(1.8)$ reduces to Ljunggren's congruence $\left(\begin{array}{c}p n \\ p r\end{array}\right) \equiv\left(\begin{array}{l}n \\ r\end{array}\right)$ $\left(\bmod p^{a_{p}}\right)(\mathrm{cf} .[\mathrm{G}])$, which is an extension of the Wolstenholme congruence $\left(\begin{array}{c}2 p \\ p\end{array}\right) \equiv 2\left(\bmod p^{a_{p}}\right)$ (i.e., $\left.\left(\begin{array}{c}2 p-1 \\ p-1\end{array}\right) \equiv 1\left(\bmod p^{a_{p}}\right)\right)$. Note also that $(1.8)$ holds for every $l \in \mathbb{N}$ if and only if we have

$$
\left(\begin{array}{c}
p n \\
p r
\end{array}\right)_{f, p^{\alpha+1}} \equiv\left(\begin{array}{l}
n \\
r
\end{array}\right)_{f, p^{\alpha}}\left(\bmod p^{a_{p}}\right)
$$

for all $f(x) \in \mathbb{Z}[x]$, where

$$
\left(\begin{array}{l}
n \\
r
\end{array}\right)_{f, p^{\alpha}}=\frac{p^{\operatorname{deg} f}}{\left\lfloor n / p^{\alpha-1}\right\rfloor !} \sum_{k \equiv r\left(\bmod p^{\alpha}\right)}(-1)^{k}\left(\begin{array}{l}
n \\
k
\end{array}\right) f\left(\frac{k-r}{p^{\alpha}}\right) \in \mathbb{Z}_{p} .
$$

(As usual, $\mathbb{Z}_{p}$ denotes the ring of $p$-adic integers.)

Concerning the right-hand side of the congruence (1.8), a Lucas-type congruence modulo $p$ was established in [SD] for $\alpha>1$ (and in [SW] for $\alpha=1)$. See also [SW] for some other congruences of Lucas' type related to combinatorial sums involving binomial coefficients.

In the next section we prove Theorem 1.1 and Corollary 1.3. On the basis of Theorem 1.1 we will deduce Theorem 1.2 in Section 3. Section 4 is devoted to the proof of Theorem 1.3.

\section{Proofs of Theorem 1.1 and Corollary 1.3}

Proof of Theorem 1.1. By a well-known property of Stirling numbers of the second kind (cf. [LW, pp. 125-126]),

$$
\begin{aligned}
\sum_{j=0}^{l}\left(\begin{array}{l}
l \\
j
\end{array}\right) a^{l-j} S(j, m) & =\sum_{j=0}^{l}\left(\begin{array}{l}
l \\
j
\end{array}\right) a^{l-j} \frac{1}{m !} \sum_{k=0}^{m}(-1)^{m-k}\left(\begin{array}{c}
m \\
k
\end{array}\right) k^{j} \\
& =\frac{1}{m !} \sum_{k=0}^{m}(-1)^{m-k}\left(\begin{array}{c}
m \\
k
\end{array}\right)(a+k)^{l}=(-1)^{m} \sum_{r=0}^{p-1} S_{r}(l),
\end{aligned}
$$

where

$$
S_{r}(l)=\frac{1}{m !} \sum_{k \equiv r(\bmod p)}(-1)^{k}\left(\begin{array}{c}
m \\
k
\end{array}\right)(a+k)^{l}
$$


Let $r \in\{0, \ldots, p-1\}$. Observe that

$$
\begin{aligned}
S_{r}(l) & =\frac{1}{m !} \sum_{k \equiv r(\bmod p)}(-1)^{k}\left(\begin{array}{c}
m \\
k
\end{array}\right) \sum_{j=0}^{l}\left(\begin{array}{l}
l \\
j
\end{array}\right) p^{j}\left(\frac{k-r}{p}\right)^{j}(a+r)^{l-j} \\
& =\sum_{j=0}^{l}\left(\begin{array}{l}
l \\
j
\end{array}\right)(a+r)^{l-j} \frac{p^{j}}{m !} \sum_{k \equiv r(\bmod p)}(-1)^{k}\left(\begin{array}{c}
m \\
k
\end{array}\right)\left(\frac{k-r}{p}\right)^{j} .
\end{aligned}
$$

By Theorem 1.0, for any $j \in \mathbb{N}$ we have

$$
\sigma_{r}(j):=\frac{p^{j}}{m !} \sum_{k \equiv r(\bmod p)}(-1)^{k}\left(\begin{array}{c}
m \\
k
\end{array}\right)\left(\frac{k-r}{p}\right)_{j} \in \mathbb{Z}_{p}
$$

and

$$
\frac{p^{j}}{m !} \sum_{k \equiv r(\bmod p)}(-1)^{k}\left(\begin{array}{c}
m \\
k
\end{array}\right)\left(\left(\frac{k-r}{p}\right)^{j}-\left(\frac{k-r}{p}\right)_{j}\right) \equiv 0(\bmod p)
$$

since the degree of $f_{j}(x)=x^{j}-(x)_{j} \in \mathbb{Z}[x]$ is smaller than $j$. Therefore,

$$
S_{r}(l) \equiv \sum_{j=0}^{l}\left(\begin{array}{l}
l \\
j
\end{array}\right)(a+r)^{l-j} \sigma_{r}(j)(\bmod p) .
$$

In view of the above, it suffices to show that

$$
\left(\begin{array}{l}
l^{\prime} \\
j
\end{array}\right)(a+r)^{l^{\prime}-j} \sigma_{r}(j) \equiv\left(\begin{array}{l}
l \\
j
\end{array}\right)(a+r)^{l-j} \sigma_{r}(j)(\bmod p)
$$

for every $j=0,1, \ldots$

Below we assume $j \in \mathbb{N}$ and $\sigma_{r}(j) \neq 0$. Then $((k-r) / p)_{j} \neq 0$ for some $0 \leq k \leq m$ with $k \equiv r(\bmod p)$, hence $m-r \geq k-r \geq p j$ and $j \leq m / p<l \leq l^{\prime}$. If $p \mid a+r$, then

$$
(a+r)^{l^{\prime}-j} \equiv 0 \equiv(a+r)^{l-j}(\bmod p) .
$$

When $p \nmid a+r$, as $l^{\prime} \equiv l(\bmod p-1)$ we have

$$
(a+r)^{l^{\prime}-j} \equiv(a+r)^{l-j}(\bmod p)
$$

by Fermat's little theorem. So it remains to show $\left(\begin{array}{c}l^{\prime} \\ j\end{array}\right) \equiv\left(\begin{array}{l}l \\ j\end{array}\right)(\bmod p)$ in the case $p \nmid a+r$.

Let $\alpha=\left\lfloor\log _{p} m\right\rfloor$. Then $p^{\alpha} \leq m<p^{\alpha+1}$ and $\delta=\delta_{p}(a, m) \leq \alpha$. Write $l=p^{\alpha-\delta} q_{0}+l_{0}$ with $q_{0} \in \mathbb{N}$ and $0 \leq l_{0}<p^{\alpha-\delta}$. For some $q \in \mathbb{N}$ we have $l^{\prime}=l+(p-1) p^{\alpha-\delta} q=p^{\alpha-\delta}\left((p-1) q+q_{0}\right)+l_{0}$. Recall that $j \leq(m-r) / p<p^{\alpha}$. Suppose $a+r \not \equiv 0(\bmod p)$. If $\delta=1$, then $j<m / p=p^{\alpha-1}$ because $m=p^{\alpha}$ and $r \neq\{-a\}_{p}=0$. Thus $j<p^{\alpha-\delta}$. With the help of the Chu-Vandermonde 
convolution identity (cf. [GKP, (5.27)]),

$$
\begin{aligned}
\left(\begin{array}{l}
l \\
j
\end{array}\right)-\left(\begin{array}{c}
l_{0} \\
j
\end{array}\right) & =\sum_{0<i \leq j}\left(\begin{array}{c}
p^{\alpha-\delta} q_{0} \\
i
\end{array}\right)\left(\begin{array}{c}
l_{0} \\
j-i
\end{array}\right) \\
& =\sum_{0<i \leq j} \frac{p^{\alpha-\delta}}{i} q_{0}\left(\begin{array}{c}
p^{\alpha-\delta} q_{0}-1 \\
i-1
\end{array}\right)\left(\begin{array}{c}
l_{0} \\
j-i
\end{array}\right) \equiv 0(\bmod p) .
\end{aligned}
$$

Similarly, $\left(\begin{array}{c}l^{\prime} \\ j\end{array}\right) \equiv\left(\begin{array}{c}l_{0} \\ j\end{array}\right)(\bmod p)$ as desired. We are done.

Proof of Corollary 1.3. Let $g$ be a primitive root modulo $p$. For any integer $h$, if $p-1 \mid h$ then $\sum_{a=1}^{p-1} a^{h} \equiv p-1 \equiv-1(\bmod p)$ by Fermat's little theorem; if $p-1 \nmid h$ then $g^{h} \not \equiv 1(\bmod p)$ and hence $\sum_{a=1}^{p-1} a^{h} \equiv 0(\bmod p)$ since

$$
\left(g^{h}-1\right) \sum_{a=1}^{p-1} a^{h}=\sum_{a=1}^{p-1}(a g)^{h}-\sum_{a=1}^{p-1} a^{h} \equiv 0(\bmod p) .
$$

In view of the above,

$$
\begin{aligned}
\sum_{a=1}^{p-1} a^{r-l} \sum_{j=0}^{l}\left(\begin{array}{l}
l \\
j
\end{array}\right) S(j, m) a^{l-j} & =\sum_{j=0}^{l}\left(\begin{array}{l}
l \\
j
\end{array}\right) S(j, m) \sum_{a=1}^{p-1} a^{r-j} \\
& \equiv-\sum_{j \equiv r(\bmod p-1)}\left(\begin{array}{l}
l \\
j
\end{array}\right) S(j, m)(\bmod p) .
\end{aligned}
$$

Similarly,

$$
\sum_{a=1}^{p-1} a^{r-l^{\prime}} \sum_{j=0}^{l^{\prime}}\left(\begin{array}{c}
l^{\prime} \\
j
\end{array}\right) S(j, m) a^{l^{\prime}-j} \equiv-\sum_{j \equiv r(\bmod p-1)}\left(\begin{array}{l}
l^{\prime} \\
j
\end{array}\right) S(j, m)(\bmod p) .
$$

Since $l^{\prime} \equiv l(\bmod p-1)$, it follows that $a^{r-l^{\prime}} \equiv a^{r-l}(\bmod p)$ for all $a=$ $1, \ldots, p-1$. Thus, applying Theorem 1.1 we immediately obtain (1.4) from the above.

3. Proof of Theorem 1.2. First we make some useful observations. Clearly

$$
m \leq \frac{n-r_{*}}{p^{\alpha}}=\frac{n-r}{p^{\alpha}}+\left\lfloor\frac{r}{p^{\alpha}}\right\rfloor<1+\left\lfloor\frac{n-r}{p^{\alpha}}\right\rfloor+\left\lfloor\frac{r}{p^{\alpha}}\right\rfloor=m+1 .
$$

Since

$$
\tau_{p}\left(\{r\}_{p^{\alpha}},\{n-r\}_{p^{\alpha}}\right)-\tau_{p}\left(\{r\}_{p^{\alpha-1}},\{n-r\}_{p^{\alpha-1}}\right)= \begin{cases}1 & \text { if } n_{*} \geq p^{\alpha} \\ 0 & \text { otherwise }\end{cases}
$$

we also have

$$
\operatorname{ord}_{p}\left(\begin{array}{l}
n_{*} \\
r_{*}
\end{array}\right)-\tau_{p}\left(\{r\}_{p^{\alpha-1}},\{n-r\}_{p^{\alpha-1}}\right)=\left\lfloor\frac{n_{*}}{p^{\alpha}}\right\rfloor=\left\lfloor\frac{n}{p^{\alpha}}\right\rfloor-m .
$$


Let $a=-\left\lfloor r / p^{\alpha}\right\rfloor, k \in\{0, \ldots, n\}$ and $k \equiv r\left(\bmod p^{\alpha}\right)$. Then

$$
\begin{aligned}
\left(\frac{k-r}{p^{\alpha}}\right)^{l} & =\left(\frac{k-r_{*}}{p^{\alpha}}+a\right)^{l}=\sum_{j=0}^{l}\left(\begin{array}{l}
l \\
j
\end{array}\right) a^{l-j}\left(\frac{k-r_{*}}{p^{\alpha}}\right)^{j} \\
& =\sum_{j=0}^{l}\left(\begin{array}{l}
l \\
j
\end{array}\right) a^{l-j} \sum_{i=0}^{j} S(j, i)\left(\frac{k-r_{*}}{p^{\alpha}}\right)_{i} \\
& =\sum_{j=0}^{l}\left(\begin{array}{l}
l \\
j
\end{array}\right) a^{l-j} \sum_{i=0}^{m} S(j, i)\left(\frac{k-r_{*}}{p^{\alpha}}\right)_{i}
\end{aligned}
$$

because for $i \geq m+1$ we have $i>\left(n-r_{*}\right) / p^{\alpha} \geq\left(k-r_{*}\right) / p^{\alpha}$ and hence $\left(\left(k-r_{*}\right) / p^{\alpha}\right)_{i}=0$.

Observe that

$$
\operatorname{ord}_{p}\left(\left\lfloor\frac{n}{p^{\alpha-1}}\right\rfloor !\right)=\left\lfloor\frac{n}{p^{\alpha}}\right\rfloor+\sum_{s>\alpha}\left\lfloor\frac{n}{p^{s}}\right\rfloor=\left\lfloor\frac{n}{p^{\alpha}}\right\rfloor+\operatorname{ord}_{p}\left(\left\lfloor\frac{n}{p^{\alpha}}\right\rfloor !\right) .
$$

If $i \in\{0, \ldots, m-1\}$, then by Theorem 1.0 and (3.1) we have

$$
\begin{gathered}
\operatorname{ord}_{p}\left(\sum_{k \equiv r_{*}\left(\bmod p^{\alpha}\right)}(-1)^{k}\left(\begin{array}{l}
n \\
k
\end{array}\right)\left(\frac{k-r_{*}}{p^{\alpha}}\right)_{i}\right) \\
\quad \geq \operatorname{ord}_{p}\left(\left\lfloor\frac{n}{p^{\alpha-1}}\right\rfloor\right)-i+\tau_{p}\left(\left\{r_{*}\right\}_{p^{\alpha-1}},\left\{n-r_{*}\right\}_{p^{\alpha-1}}\right) \\
>\operatorname{ord}_{p}\left(\left\lfloor\frac{n}{p^{\alpha}}\right\rfloor !\right)+\left\lfloor\frac{n}{p^{\alpha}}\right\rfloor-m+\tau_{p}\left(\{r\}_{p^{\alpha-1}},\{n-r\}_{p^{\alpha-1}}\right) \\
\quad=\operatorname{ord}_{p}\left(\left\lfloor\frac{n}{p^{\alpha}}\right\rfloor !\left(\begin{array}{c}
n_{*} \\
r_{*}
\end{array}\right)\right) .
\end{gathered}
$$

Therefore,

$$
\begin{aligned}
& \frac{1}{\left\lfloor n / p^{\alpha}\right\rfloor !\left(\begin{array}{c}
n_{*} \\
r_{*}
\end{array}\right)} \sum_{k \equiv r\left(\bmod p^{\alpha}\right)}(-1)^{k}\left(\begin{array}{l}
n \\
k
\end{array}\right)\left(\frac{k-r}{p^{\alpha}}\right)^{l} \\
& \quad \equiv \sum_{j=0}^{l}\left(\begin{array}{l}
l \\
j
\end{array}\right) \frac{S(j, m) a^{l-j}}{\left\lfloor n / p^{\alpha}\right\rfloor !\left(\begin{array}{c}
n_{*} \\
r_{*}
\end{array}\right)} \sum_{k \equiv r\left(\bmod p^{\alpha}\right)}(-1)^{k}\left(\begin{array}{l}
n \\
k
\end{array}\right)\left(\frac{k-r_{*}}{p^{\alpha}}\right)_{m}(\bmod p) .
\end{aligned}
$$

In light of Corollary 1.1 , it remains to show that $S \equiv(-1)^{l+r_{*}}(\bmod p)$, where

$$
S=\frac{1}{\left\lfloor n / p^{\alpha}\right\rfloor !\left(\begin{array}{c}
n_{*} \\
r_{*}
\end{array}\right)} \sum_{k \equiv r_{*}\left(\bmod p^{\alpha}\right)}(-1)^{k}\left(\begin{array}{l}
n \\
k
\end{array}\right)\left(\frac{k-r_{*}}{p^{\alpha}}\right)_{m} .
$$


If $k \in\{0, \ldots, n\}, k \equiv r_{*}\left(\bmod p^{\alpha}\right)$ and $\left(\left(k-r_{*}\right) / p^{\alpha}\right)_{m} \neq 0$, then

$$
m \leq \frac{k-r_{*}}{p^{\alpha}} \leq \frac{n-r_{*}}{p^{\alpha}}<m+1
$$

and hence $k=m p^{\alpha}+r_{*}$. So

$$
\begin{aligned}
S & =\frac{(-1)^{m p^{\alpha}+r_{*}}}{\left\lfloor n / p^{\alpha}\right\rfloor !\left(\begin{array}{c}
n_{*} \\
r_{*}
\end{array}\right)}\left(\begin{array}{c}
n \\
m p^{\alpha}+r_{*}
\end{array}\right)(m)_{m} \\
& =\frac{(-1)^{m p^{\alpha}+r_{*}} m !}{\left(m+\left\lfloor n_{*} / p^{\alpha}\right\rfloor\right) !} \cdot \frac{\left(\begin{array}{l}
m p^{\alpha}+n_{*} \\
m p^{\alpha}+r_{*}
\end{array}\right)}{\left(\begin{array}{l}
n_{*} \\
r_{*}
\end{array}\right)} .
\end{aligned}
$$

Clearly

$$
\begin{aligned}
\frac{\left(\begin{array}{c}
m p^{\alpha}+n_{*} \\
m p^{\alpha}+r_{*}
\end{array}\right)}{\left(\begin{array}{l}
n_{*} \\
r_{*}
\end{array}\right)} & =\frac{\left(m p^{\alpha}+n_{*}\right) ! /\left(m p^{\alpha}+r_{*}\right) !}{n_{*} ! / r_{*} !} \\
& =\prod_{0<i \leq n_{*}}\left(1+m \frac{p^{\alpha}}{i}\right) / \prod_{0<j \leq r_{*}}\left(1+m \frac{p^{\alpha}}{j}\right) .
\end{aligned}
$$

Thus, if $n_{*}<p^{\alpha}$ then

$$
\frac{\left(\begin{array}{c}
m p^{\alpha}+n_{*} \\
m p^{\alpha}+r_{*}
\end{array}\right)}{\left(\begin{array}{l}
n_{*} \\
r_{*}
\end{array}\right)} \equiv 1(\bmod p)
$$

while if $n_{*} \geq p^{\alpha}$ then $\left\lfloor n_{*} / p^{\alpha}\right\rfloor=1$ and

$$
\begin{aligned}
\frac{\left(\begin{array}{c}
m p^{\alpha}+n_{*} \\
m p^{\alpha}+r_{*}
\end{array}\right)}{(m+1)\left(\begin{array}{c}
n_{*} \\
r_{*}
\end{array}\right)} & =\prod_{\substack{0<i \leq n_{*} \\
i \neq p^{\alpha}}}\left(1+m \frac{p^{\alpha}}{i}\right) / \prod_{0<j \leq r_{*}}\left(1+m \frac{p^{\alpha}}{j}\right) \\
& \equiv 1(\bmod p) .
\end{aligned}
$$

Therefore,

$$
S \equiv(-1)^{m p^{\alpha}+r_{*}} \equiv(-1)^{m+r_{*}} \equiv(-1)^{l+r_{*}}(\bmod p) .
$$

This concludes the proof of Theorem 1.2.

4. Proof of Theorem 1.3. For $i, k \in \mathbb{N}$ let $\delta_{i, k}$ be the Kronecker symbol. Since

$$
\delta_{i, k}=\left(\begin{array}{c}
k \\
i
\end{array}\right) \sum_{j \geq i}(-1)^{j-i}\left(\begin{array}{c}
k-i \\
j-i
\end{array}\right)=\sum_{j \geq i}(-1)^{j-i}\left(\begin{array}{l}
k \\
j
\end{array}\right)\left(\begin{array}{l}
j \\
i
\end{array}\right)
$$


we have

$$
\begin{aligned}
(-1)^{(p-1) r} & \sum_{k \equiv r\left(\bmod p^{\alpha}\right)}(-1)^{k}\left(\begin{array}{l}
p n \\
p k
\end{array}\right)\left(\frac{k-r}{p^{\alpha-1}}\right)^{l} \\
& =\sum_{k \equiv r\left(\bmod p^{\alpha}\right)}(-1)^{p k}\left(\begin{array}{c}
p n \\
p k
\end{array}\right)\left(\frac{k-r}{p^{\alpha-1}}\right)^{l} \\
& =\sum_{k=0}^{n}(-1)^{p k}\left(\begin{array}{c}
p n \\
p k
\end{array}\right) \sum_{i \equiv r\left(\bmod p^{\alpha}\right)}\left(\frac{i-r}{p^{\alpha-1}}\right)^{l} \delta_{i, k} \\
& =\sum_{k=0}^{n}(-1)^{p k}\left(\begin{array}{c}
p n \\
p k
\end{array}\right) \sum_{i \equiv r\left(\bmod p^{\alpha}\right)}\left(\frac{i-r}{p^{\alpha-1}}\right)^{l} \sum_{j \geq i}(-1)^{j-i}\left(\begin{array}{l}
k \\
j
\end{array}\right)\left(\begin{array}{l}
j \\
i
\end{array}\right) \\
& =\sum_{j=0}^{n}(-1)^{j} C_{n, j} \sum_{i \equiv r\left(\bmod p^{\alpha}\right)}(-1)^{i}\left(\begin{array}{l}
j \\
i
\end{array}\right)\left(\frac{i-r}{p^{\alpha-1}}\right)^{l},
\end{aligned}
$$

where

$$
C_{n, j}=\sum_{k=0}^{n}(-1)^{p k}\left(\begin{array}{c}
p n \\
p k
\end{array}\right)\left(\begin{array}{l}
k \\
j
\end{array}\right)=\sum_{k \equiv 0(\bmod p)}(-1)^{k}\left(\begin{array}{c}
p n \\
k
\end{array}\right)\left(\begin{array}{c}
k / p \\
j
\end{array}\right) .
$$

As $C_{n, n}=(-1)^{p n}$, by the above

$$
\begin{aligned}
(-1)^{(p-1) r} \sum_{k \equiv r\left(\bmod p^{\alpha}\right)}(-1)^{k}\left(\begin{array}{c}
p n \\
p k
\end{array}\right)\left(\frac{k-r}{p^{\alpha-1}}\right)^{l} & \\
& -(-1)^{(p-1) n} \sum_{i \equiv r\left(\bmod p^{\alpha}\right)}(-1)^{i}\left(\begin{array}{c}
n \\
i
\end{array}\right)\left(\frac{i-r}{p^{\alpha-1}}\right)^{l} \\
= & \sum_{0 \leq j<n}(-1)^{j} C_{n, j} \sum_{i \equiv r\left(\bmod p^{\alpha}\right)}(-1)^{i}\left(\begin{array}{l}
j \\
i
\end{array}\right)\left(\frac{i-r}{p^{\alpha-1}}\right)^{l} .
\end{aligned}
$$

Note that $(-1)^{(p-1) n} \equiv(-1)^{(p-1) r}\left(\bmod p^{a_{p}}\right)$. In view of Theorem 1.0 ,

$$
\operatorname{ord}_{p}\left(\sum_{i \equiv r\left(\bmod p^{\alpha}\right)}(-1)^{i}\left(\begin{array}{l}
j \\
i
\end{array}\right)\left(\frac{i-r}{p^{\alpha-1}}\right)^{l}\right) \geq \operatorname{ord}_{p}\left(\left\lfloor\frac{j}{p^{\alpha-1}}\right\rfloor !\right)=\sum_{s=\alpha}^{\infty}\left\lfloor\frac{j}{p^{s}}\right\rfloor .
$$

So it suffices to show that

$$
\operatorname{ord}_{p}\left(C_{n, j}\right) \geq a_{p}+\operatorname{ord}_{p}\left(\left\lfloor\frac{n}{p^{\alpha-1}}\right\rfloor !\right)-\sum_{s=\alpha}^{\infty}\left\lfloor\frac{j}{p^{s}}\right\rfloor=a_{p}+\sum_{s=\alpha}^{\infty}\left(\left\lfloor\frac{n}{p^{s}}\right\rfloor-\left\lfloor\frac{j}{p^{s}}\right\rfloor\right)
$$

for any $j \in \mathbb{N}$ with $j<n$. 
Fix a nonnegative integer $j<n$. In light of Theorem 1.0,

$$
\operatorname{ord}_{p}\left(j ! C_{n, j}\right) \geq \operatorname{ord}_{p}\left(\left\lfloor\frac{p n}{p^{1-1}}\right\rfloor !\right)-j=\sum_{s=0}^{\infty}\left\lfloor\frac{n}{p^{s}}\right\rfloor-j .
$$

By Lemma 3.2 of [SD] and its proof, $C_{n, j}$ is congruent to

$$
\sum_{k=0}^{n}(-1)^{k}\left(\begin{array}{l}
n \\
k
\end{array}\right)\left(\begin{array}{l}
k \\
j
\end{array}\right)=(-1)^{j}\left(\begin{array}{l}
n \\
j
\end{array}\right) \sum_{k \geq j}(-1)^{k-j}\left(\begin{array}{l}
n-j \\
k-j
\end{array}\right)=0
$$

modulo $p^{2 \operatorname{ord}_{p}(n)+a_{p}}$. In the case $p>3$, by Jacobsthal's result (cf. [G]), if $k \in\{1, \ldots, n\}$, then

$$
\left(\begin{array}{l}
p n \\
p k
\end{array}\right) /\left(\begin{array}{l}
n \\
k
\end{array}\right)=1+p^{3} n k(n-k) q_{k}
$$

for some $q_{k} \in \mathbb{Z}_{p}$, and hence

$$
\begin{aligned}
\left(\begin{array}{l}
p n \\
p k
\end{array}\right)-\left(\begin{array}{l}
n \\
k
\end{array}\right) & =\left(\begin{array}{l}
n \\
k
\end{array}\right) p^{3} n k(n-k) q_{k} \\
& =p^{3} n^{2}(n-1)\left(\begin{array}{l}
n-2 \\
k-1
\end{array}\right) q_{k} .
\end{aligned}
$$

So we also have $\operatorname{ord}_{p}\left(C_{n, j}\right) \geq \operatorname{ord}_{p}(n-1)+3$ when $p>3$. These facts will be used in the following discussion.

CASE $1: n-j \geq a_{p}$. In this case,

$$
\begin{aligned}
\operatorname{ord}_{p}\left(C_{n, j}\right) & \geq \sum_{s=0}^{\infty}\left\lfloor\frac{n}{p^{s}}\right\rfloor-j-\operatorname{ord}_{p}(j !) \\
& =\sum_{s=0}^{\infty}\left(\left\lfloor\frac{n}{p^{s}}\right\rfloor-\left\lfloor\frac{j}{p^{s}}\right\rfloor\right) \\
& =n-j+\sum_{s=1}^{\infty}\left(\left\lfloor\frac{n}{p^{s}}\right\rfloor-\left\lfloor\frac{j}{p^{s}}\right\rfloor\right) \\
& \geq a_{p}+\sum_{s=\alpha}^{\infty}\left(\left\lfloor\frac{n}{p^{s}}\right\rfloor-\left\lfloor\frac{j}{p^{s}}\right\rfloor\right) .
\end{aligned}
$$

CASE 2: $0<n-j<a_{p} \leq 3$, and $p \mid n$ or $j \neq n-2$. If $\beta=\operatorname{ord}_{p}(n)>0$, then $n-j<a_{p}<p \leq p^{\beta}$ and hence

$$
\left\lfloor\frac{n}{p^{\beta+1}}\right\rfloor=\left\lfloor\frac{n / p^{\beta}}{p}\right\rfloor=\left\lfloor\frac{n / p^{\beta}-1}{p}\right\rfloor=\left\lfloor\frac{\left\lfloor j / p^{\beta}\right\rfloor}{p}\right\rfloor=\left\lfloor\frac{j}{p^{\beta+1}}\right\rfloor,
$$


therefore

$$
\begin{aligned}
\sum_{s=\alpha}^{\infty}\left(\left\lfloor\frac{n}{p^{s}}\right\rfloor-\left\lfloor\frac{j}{p^{s}}\right\rfloor\right) & =\sum_{\alpha \leq s \leq \beta}\left(\frac{n}{p^{s}}-\left\lfloor\frac{j}{p^{s}}\right\rfloor\right) \\
& \leq \sum_{\alpha \leq s \leq \beta} 1<2 \beta=2 \operatorname{ord}_{p}(n) \\
& \leq \operatorname{ord}_{p}\left(C_{n, j}\right)-a_{p} .
\end{aligned}
$$

When $\beta=\operatorname{ord}_{p}(n)=0$ (i.e., $p \nmid n$ ) and $j=n-1$, we have

$$
\sum_{s=\alpha}^{\infty}\left(\left\lfloor\frac{n}{p^{s}}\right\rfloor-\left\lfloor\frac{j}{p^{s}}\right\rfloor\right)=0=2 \operatorname{ord}_{p}(n) \leq \operatorname{ord}_{p}\left(C_{n, j}\right)-a_{p} .
$$

CASE $3: n-j=2<a_{p}$ and $p \nmid n$. In this case, $a_{p}=3<p$ and

$$
\begin{aligned}
& \operatorname{ord}_{p}\left(C_{n, j}\right)-a_{p} \geq \operatorname{ord}_{p}(n-1) \\
& \geq \sum_{\alpha \leq s \leq \operatorname{ord}_{p}(n-1)}\left(\frac{n-1}{p^{s}}-\left\lfloor\frac{n-2}{p^{s}}\right\rfloor\right) \\
& =\sum_{s=\alpha}^{\infty}\left(\left\lfloor\frac{n-1}{p^{s}}\right\rfloor-\left\lfloor\frac{n-2}{p^{s}}\right\rfloor\right) \\
& =\sum_{s=\alpha}^{\infty}\left(\left\lfloor\frac{n}{p^{s}}\right\rfloor-\left\lfloor\frac{j}{p^{s}}\right\rfloor\right) .
\end{aligned}
$$

Combining the above we have completed the proof of Theorem 1.3.

\section{References}

[C] L. Carlitz, Congruences for generalized Bell and Stirling numbers, Duke Math. J. 22 (1955), 193-205.

[DS] D. M. Davis and Z. W. Sun, A number-theoretic approach to homotopy exponents of SU(n), J. Pure Appl. Algebra 209 (2007), 57-69.

[D] L. E. Dickson, History of the Theory of Numbers, Vol. I, AMS Chelsea Publ., 1999 .

[GKP] R. Graham, D. E. Knuth and O. Patashnik, Concrete Mathematics, AddisonWesley, New York, 1989.

[G] A. Granville, Arithmetic properties of binomial coefficients. I. Binomial coefficients modulo prime powers, in: Organic Mathematics (Burnaby, BC, 1995), CMS Conf. Proc. 20, Amer. Math. Soc., Providence, RI, 1997, 253-276.

[IR] K. Ireland and M. Rosen, A Classical Introduction to Modern Number Theory, 2nd ed., Grad. Texts in Math. 84, Springer, New York, 1990.

[K] Y. H. H. Kwong, Minimum periods of $S(n, k)$ modulo $M$, Fibonacci Quart. 27 (1989), 217-221.

[LW] J. H. van Lint and R. M. Wilson, A Course in Combinatorics, 2nd ed., Cambridge Univ. Press, Cambridge, 2001. 
[NW] A. Nijenhuis and H. S. Wilf, Periodicities of partition functions and Stirling numbers modulo p, J. Number Theory 25 (1987), 308-312.

[S] Z. W. Sun, On the sum $\sum_{k \equiv r(\bmod m)}\left(\begin{array}{l}n \\ k\end{array}\right)$ and related congruences, Israel J. Math. 128 (2002), 135-156.

[SD] Z. W. Sun and D. M. Davis, Combinatorial congruences modulo prime powers, Trans. Amer. Math. Soc., in press; http://arxiv.org/abs/math.NT/0508087.

[ST] Z. W. Sun and R. Tauraso, Congruences for sums of binomial coefficients, J. Number Theory, to appear; http://arxiv.org/abs/math.NT/0502187.

[SW] Z. W. Sun and D. Wan, Lucas-type congruences for cyclotomic $\psi$-coefficients, Int. J. Number Theory, to appear; http://arxiv.org/abs/math.NT/0512012.

Department of Mathematics

Nanjing University

Nanjing 210093

People's Republic of China

E-mail: zwsun@nju.edu.cn

Web: http://pweb.nju.edu.cn/zwsun

Received on 9.3.2006

and in revised form on 6.12.2006 